\title{
Restoration of radiation therapy-induced salivary gland dysfunction in mice by post therapy IGF-1 administration
}

\author{
Oliver Grundmann, Jamia L Fillinger, Kerton R Victory, Randy Burd, Kirsten H Limesand
}

\begin{abstract}
Background: Radiotherapy for head and neck cancer results in severe and chronic salivary gland dysfunction in most individuals. This results in significant side effects including xerostomia, dysphagia, and malnutrition which are linked to significant reductions in patients' quality of life. Currently there are few xerostomia treatment approaches that provide long-term results without significant side effects. To address this problem we investigated the potential for post-therapeutic IGF-1 to reverse radiation-induced salivary gland dysfunction.

Methods: FVB mice were treated with targeted head and neck radiation and significant reductions in salivary function were confirmed 3 days after treatment. On days 4-8 after radiation, one group of mice was injected intravenously with IGF-1 while a second group served as a vehicle control. Stimulated salivary flow rates were evaluated on days 30, 60, and 90 and histological analysis was performed on days 9, 30, 60, and 90.
\end{abstract}

Results: Irradiated animals receiving vehicle injections have 40-50\% reductions in stimulated salivary flow rates throughout the entire time course. Mice receiving injections of IGF-1 have improved stimulated salivary flow rates 30 days after treatment. By days 60-90, IGF-1 injected mice have restored salivary flow rates to unirradiated control mice levels. Parotid tissue sections were stained for amylase as an indicator of functioning acinar cells and significant reductions in total amylase area are detected in irradiated animals compared to unirradiated groups on all days. Post-therapeutic injections of IGF-1 results in increased amylase-positive acinar cell area and improved amylase secretion. Irradiated mice receiving IGF-1 show similar proliferation indices as untreated mice suggesting a return to tissue homeostasis.

Conclusions: Post-therapeutic IGF-1 treatment restores salivary gland function potentially through normalization of cell proliferation and improved expression of amylase. These findings could aid in the rational design of therapy protocols or drugs for the treatment of radiation-induced salivary gland dysfunction in patients who have completed their anti-cancer therapies.

\section{Background}

The treatment of head and neck cancer commonly involves fractionated radiation therapy which results in significant side effects including xerostomia, dysphagia, and increased infection rates in most patients [1-3]. Although attempts are made to limit radiation exposure to spare normal tissues, the close proximity of the salivary glands to the treatment field leads to impairment of physiological function and secondary side effects that impact quality of life for patients [4]. A reduction of

\footnotetext{
* Correspondence: limesank@u.arizona.edu

Department of Nutritional Sciences, University of Arizona, Tucson, AZ, USA
}

saliva flow rates by $50-60 \%$ with accompanying changes in saliva composition ensues during the first week of continued radiation therapy which has been associated with a loss of acinar cells and glandular shrinkage $[5,6]$. Serous acinar cells of the parotid gland are the main contributor of protein and water to the composition of saliva $[7,8]$. It has been shown in humans that radiationinduced defects in amylase production by the parotid acinar cells lead to significant decreases in amylase concentration in stimulated saliva [9]. These immediate pathophysiological and structural changes may contribute to the development of chronic symptoms such as malnutrition, mucositis, and permanent reduction in
() Biomed Central

(c) 2010 Grundmann et al; licensee BioMed Central Ltd. This is an Open Access article distributed under the terms of the Creative Commons Attribution License (http://creativecommons.org/licenses/by/2.0), which permits unrestricted use, distribution, and reproduction in any medium, provided the original work is properly cited. 
saliva flow rates based on acinar cell attrition and replacement with fibrotic tissue [10-12].

Current treatment approaches focus mainly on preventing radiation-induced xerostomia. The only preventative therapy currently available is amifostine which is linked to unfavorable side effects such as hypotension, vomiting, and allergic reaction with a high discontinuation rate [13]. A promising drug for xerostomia prevention is tempol, which is currently being evaluated in clinical trials [14]. Since reductions in salivary flow rates are believed to be the cause for many of the secondary complications, palliative therapy with cholinergic agonists such as pilocarpine and cevimeline have been proposed to stimulate salivary flow $[15,16]$. However, these treatments have transient efficacy and limited success due to side effects. Promising research has been conducted in recent years that have the potential to affect the development of new treatment approaches for salivary gland dysfunction including growth factors, gene transfer, artificial salivary glands, and stem cell transplantation (reviewed in [17]).

A number of animal models have been utilized in order to understand the sensitivity of the salivary glands to therapeutic radiation (reviewed in [17]). Consistent with the human studies, all of these models demonstrate the acute and chronic loss of function; however the mechanisms responsible for the alterations in glandular physiology are debated. The mechanism of loss of acinar cell area seems to involve an apoptotic process which appears to be mediated through a p53-dependent pathway $[18,19]$. Transgenic mice expressing a constitutively activated mutant of Akt1 (myr-Akt1) suppressed radiation-induced apoptosis in vitro and in vivo by regulating the activation of p53 [18]. The use of intravenous injection of recombinant IGF-1 before radiation exposure has been shown to activate endogenous Akt in the salivary glands and suppress radiation-induced apoptosis [16]. Importantly, the suppression of apoptosis correlated with improved salivary function following radiation treatment.

Levels of radiation-induced apoptosis in mouse models appear to be dose dependent; however, the reductions in salivary flow rate are similar regardless of radiation dose $[16,18-20]$. Salivary glands present with significantly elevated levels of apoptotic cells within the first 24 hours after exposure to a single dose of ionizing radiation of which the majority are acinar cells [16,18-20]. In animal models of radiation-induced damage, reductions in salivary flow rates have been reported within 3 days posttreatment and persist out to one year (reviewed in [17]). In contrast to clinical studies, little data is available regarding the recovery from radiation-induced salivary gland dysfunction in animal models. Because apoptosis has been reported to occur initially following radiation in parotid salivary glands, the rate of acinar cell proliferation may provide further insight into restoration of radiation-induced tissue damage that leads to normal salivary flow rates in mice treated with post therapy IGF-1. The current study investigates the relationship between a single dose radiation exposure (5 Gy) and the ability of post radiation therapy administration of recombinant IGF-1 to restore salivary gland function.

\section{Methods}

\section{Mice}

Female FVB mice between 4-5 weeks old, obtained from Taconic (Taconic, Hudson, NY) were used for all experiments. Animals were maintained on a $12 \mathrm{~h}$ light/ dark cycle in accordance with the protocols approved by the University of Arizona Institutional Animal Care and Use Committee (IACUC).

\section{Radiation Treatment}

Mice were anesthetized intraperitoneally with avertin (tribromoethanol) in a dose of 0.4 to $0.6 \mathrm{mg} / \mathrm{kg}$ prior to radiation treatment. Mice were then irradiated with a single fraction of radiation (5 Gy) to the head and neck region (Cobalt-60 Teletherapy unit from Atomic Energy of Canada Ltd Theratron-80) as previously described (day 0; figure 1A) [19]. This results in irradiation of the submandibular, sublingual, parotid, and minor salivary glands. The 5 Gy radiation dose was chosen based on our previous work demonstrating the dose caused elevated levels of p53 protein, activation of apoptosis, absence of cell cycle arrest, and loss of salivary function $[18,19,21,22]$. The rest of the body (below clavicle) was shielded with $>6 \mathrm{~mm}$ thick lead which blocked $\sim 75 \%$ of the radiation to avoid systemic effects of radiation. Radiation dosimetry calculations and maintenance of the cobalt source are conducted by the Experimental Radiation Shared Service of the Arizona Cancer Center. Day 3 stimulated salivary flow rates were used to confirm significant reductions due to radiation exposure (figure 1B) and then all animals were randomized into final treatment groups. A sub-group of animals were then injected with $5 \mu$ g recombinant IGF-1 (GroPrep, Adelaide, Australia) for five consecutive days (day 4-8; figure $1 \mathrm{~A}$ ) or injected with vehicle (PBS+BSA). FVB mice were injected intravenously through the tail vein with $5 \mu \mathrm{g}$ recombinant IGF-1 (total volume of $100 \mu \mathrm{l}$ ) on days 4 through 8 ( $\sim 24$ hours apart) after radiation treatment as previously described [16]. This dose of IGF-1 was chosen because it induces maximal activation of signal transduction pathways in the salivary gland and suppresses radiation-induced loss of salivary gland function [16]. Animals were maintained and treated in accordance with protocols approved by the University of Arizona IACUC. 


\section{(A) Experimental set-up}

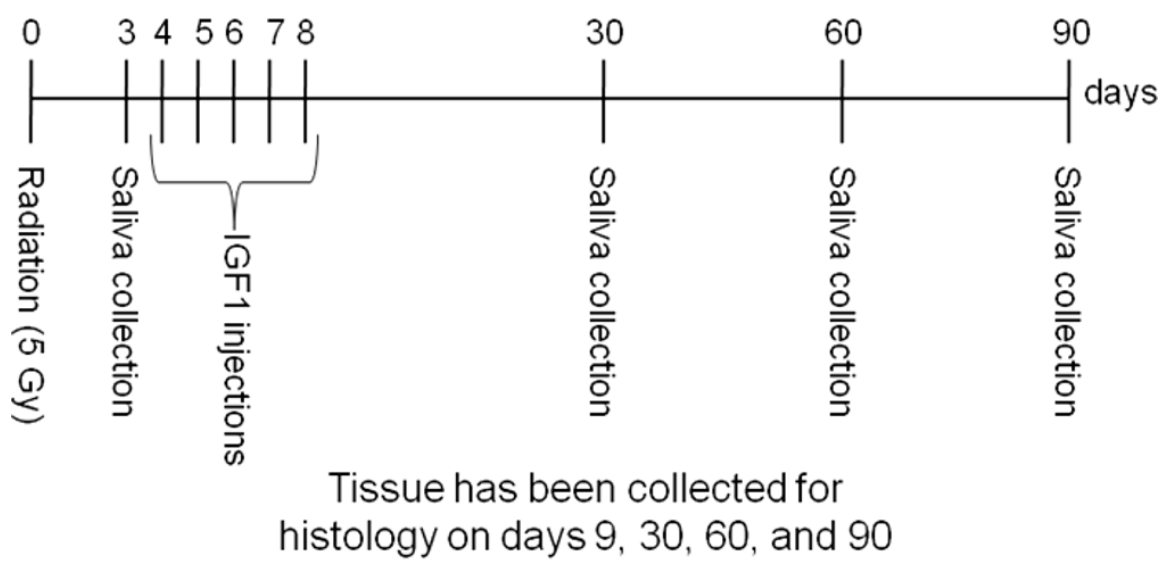

(B) 3 days

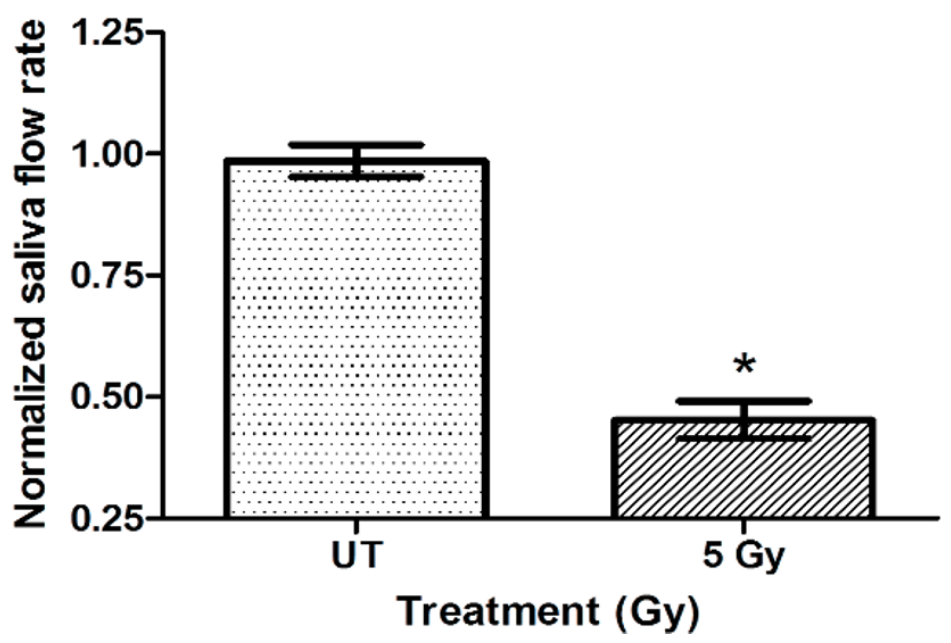

Figure 1 Experimental setting (A) and reduction in salivary flow rates on day $\mathbf{3}$ following radiation (B). The head and neck region of FVB mice was exposed to a single dose of 5 Gy radiation. Stimulated salivary flow rates were determined as described in the materials and methods section on day 3 with 81 animals in the untreated (UT) and 72 animals in the treated group. Significant differences $(p<0.05)$ were determined using an ANOVA followed by a Bonferroni test. $\left(^{*}\right)$ in figure 1B over 5Gy treatment group indicates significant differences between groups.

\section{Saliva Collection}

FVB mice were injected i.p. with carbachol $(0.25 \mathrm{mg} / \mathrm{kg}$ body weight) as previously described [18] and saliva was collected for $5 \mathrm{~min}$. on days 3, 9, 30, 60, and 90 after radiation treatments. Total saliva was collected by vacuum aspiration from 10-23 mice per treatment group as previously described [23] on ice immediately following carbachol injection into pre-weighed tubes and stored at $-80^{\circ} \mathrm{C}$. Total proteins present in the collected saliva from 7-8 mice were analyzed by resolving $25 \mu \mathrm{g}$ total protein on a $10 \%$ SDS-PAGE gel, transferred to Immunobilon membrane (Millipore Corporation,
Bedford, MA), and immunoblotted with anti-amylase (Sigma-Aldrich, St. Louis, MO). Secondary antibody was conjugated with horseradish peroxidase (anti-HRP conjugated rabbit antibody; Bio-Rad, Hercules, CA) and enhanced chemiluminescence lighting (Pierce Chemical Company, Rockford, IL) was used according to the manufacturer's instructions to detect salivary amylase.

\section{Histology}

Following excision, tissues were immediately fixed in $10 \%$ neutral buffered formalin for 24 hours, transferred to $70 \%$ ethanol, and embedded in paraffin. Sections of 
all major salivary glands were cut to $4 \mu \mathrm{m}$ thickness and processed for standard staining with hematoxylin and eosin by the Histology Service Laboratory in the Department of Cell Biology and Anatomy at the University of Arizona.

\section{Quantification of acinar cell area}

Tissue sections from mice (3-8 mice per group) treated with targeted head and neck radiation were stained for amylase (Sigma-Aldrich, St. Louis, MO) as previously described [24]. Briefly, slides were rehydrated and antigen retrieval performed as described for PCNA. Slides were blocked with $0.5 \%$ NEN at room temperature for 1 hour then incubated in anti-amylase primary antibody (1:500) overnight at $4^{\circ} \mathrm{C}$. On day 2 , slides were washed and incubated in anti-rabbit Cy2-conjugated secondary antibody (1:500) at room temperature for 1 hour, counterstained with DAPI, then mounted with $50 \%$ glycerol in $10 \mathrm{mM}$ Tris- $\mathrm{HCl}$. Fluorescent images were visualized on a Leica DM5500 Microscope System and digitally captured with a Spot Pursuit 4 Megapixel CCD camera (Diagnostic Instruments, Inc., Sterling Heights, MI). A figure insert shows a representative image demonstrating the selective staining for acinar cell area. Morphometric analysis was performed with ImagePro 7.0 software (Media Cybernetics, Silver Spring, MD). Positive areas were determined for 25 fields of view $\left(F O V=0.39 \mathrm{~mm}^{2}\right)$ and we were able to obtain a coefficient of variation of $<7 \%$, which did not improve with greater numbers of observations per section. Amylase-positive cell area was collected throughout both parotid glands of each mouse on days 30,60 , and 90 following radiation. Data are expressed as the percentage of amylase positive area to the total area of the gland and the threshold fluorescence range was equivalent for all slides imaged.

\section{Quantification of amylase protein}

The collected saliva samples from day 30 were evaluated for total amylase protein using the Bio-Rad Experion System (3-8 mice per group). Samples were loaded into a primed Experion Chip and run using the Experion Software Protein 260 Assay. Proteins of different molecular weights were visualized as bands in each sample. Analysis was done from bands located at or around the molecular weight of amylase $(54 \mathrm{kDa})$. Percent total amylase was calculated using the Experion software and graphed for each treatment group.

\section{Quantification of proliferation}

For evaluation of acinar and ductal cell proliferation, unstained tissue sections were processed for anti-PCNA (proliferating cell nuclear antigen, Santa Cruz Biotechnology Inc., Santa Cruz, CA) immunohistochemistry 9, 30,60 , and 90 days after treatment as previously described [18]. Briefly, slides were heated to $37^{\circ} \mathrm{C}$ for 30 min. and rehydrated in histoclear, graded alcohols, and distilled water washes. Nonspecific peroxidase activity was quenched with $0.3 \% \mathrm{H}_{2} \mathrm{O}_{2}$. For antigen retrieval, slides were placed in citrate buffer $(\mathrm{pH}$ 6.0) and heated in a microwave oven twice for $5 \mathrm{~min}$, and allowed to cool for $20 \mathrm{~min}$. After washes, the slides were treated according to manufacturer's instructions (Vectastain Elite ABC kit, PK-6101, Vector Laboratories Inc., Burlingame, CA). Color development was achieved with Biogenex DAB incubation for 6 to 8 minutes. Slides were lightly counterstained with Gill's hematoxylin, dehydrated, and mounted in Permount. Tissue sections were observed by standard light microscopy and photomicrographs were taken with a Leica DM5500 with a 4 megapixel Pursuit camera. Positive acinar cells were counted separately from positive ductal cells due to proposed progenitor cells present within the ductal cell network [25]. Individual means for quantification of PCNA positive acinar and ductal cells were determined by averaging the number of positive cells/total number of cells from a minimum of three fields of view/animal (3-5 mice per group; total cells counted ranged from 3,000 to 6,000 per mouse).

\section{Statistics}

Comparison of PCNA and acinar area data, amylase protein content on day 30, as well as normalized saliva flow rates was accomplished by a one-way ANOVA followed by a post-hoc Bonferroni multiple-comparison test. Saliva flow rates were normalized to the respective untreated FVB group for days 3, 30, 60, and 90. Statistical analysis and graphical generation of data were done using GraphPad Prism software (version 5.0, San Diego, CA).

\section{Results}

Stimulated salivary flow rates are restored in mice receiving post therapy IGF-1

FVB mice exposed to a single radiation dose exhibit significant $(P<0.05$, one-way ANOVA) reductions in salivary function (decreased $43-50 \%$ ) throughout the time course (figure 2A, B, and 2C) consistent with previously published studies in other animal models [12,26,27]. In contrast, injection with IGF-1 on days 4-8 following radiation increased salivary flow rates to $72 \%$ of the untreated control on day 30 compared to irradiated FVB mice (figure 2A). Further increases in salivary flow rates on days 60 and 90 in irradiated mice injected with IGF-1 result in return to untreated flow rates (93 and $81 \%$ of untreated controls, figure $2 \mathrm{~B}$ and $2 \mathrm{C}$ ). Administration of recombinant IGF-1 to unirradiated FVB mice on days 4-8 did not show significant changes in salivary flow rates at any of the time points evaluated $(85-100 \%$ of untreated controls) indicating that IGF-1 does not 

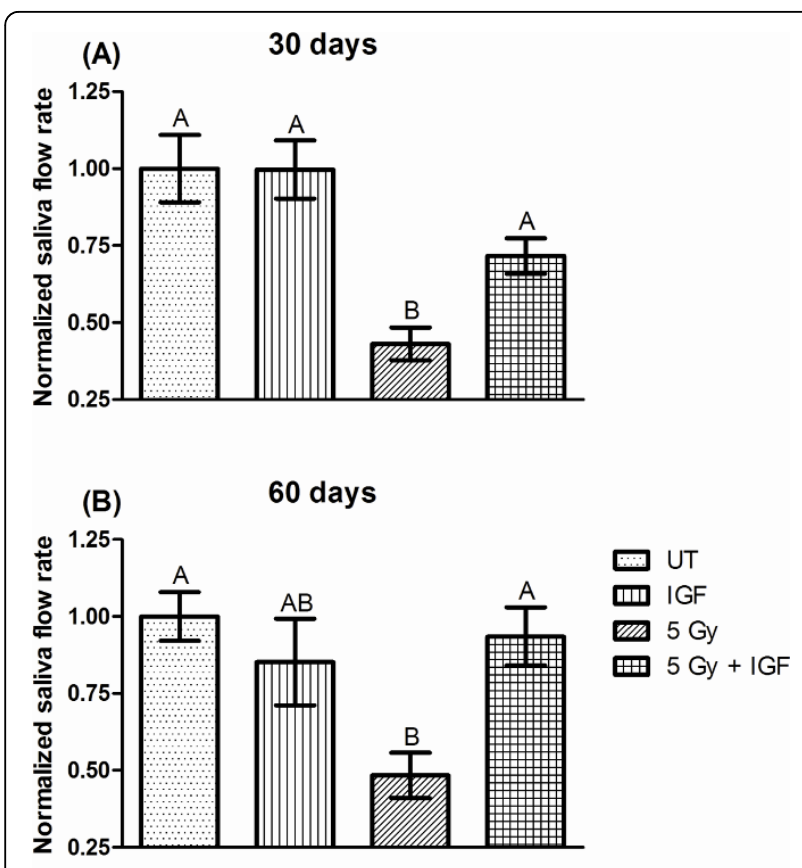

(C) 90 days

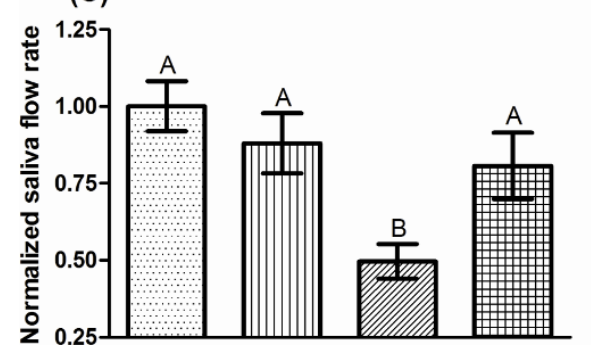

Figure 2 Salivary gland dysfunction is reversed in mice receiving post therapy IGF-1. The head and neck region of FVB mice was exposed to a single 5 Gy radiation dose and mice received injections of IGF-1 or vehicle on days 4-8 as depicted in figure $1 \mathrm{~A}$. Stimulated salivary flow rates were determined as described in the materials and methods section on days 30 (A), 60 (B), and 90 (C). Irradiated flow rates were normalized to corresponding unirradiated controls after the radiation treatment. The graph represents the mean and standard error of the mean of all data from 10-23 mice per treatment group. Significant differences ( $p<0.05$ ) were determined using an ANOVA followed by a post-hoc Bonferroni multiple-comparison test. Treatment groups with the same letters are not significantly different from each other within the same day.

affect glandular function in unstressed environments. These functional data suggest that post therapy IGF-1 can alter the response of salivary glands to radiation damage leading to restoration of function.

Post therapy IGF-1 restores functional acinar cell area and amylase secretion in irradiated mice

On all days, a consistent $14-24 \%$ reduction in amylasepositive cell area is measured for FVB mice irradiated with a single 5 Gy dose that received vehicle injections. This reduction was significantly different from untreated FVB, IGF-1 injected, and irradiated mice receiving post therapy IGF-1 $(P<0.05$, one-way ANOVA). The acinar cell areas for untreated FVB and IGF-1 injected mice are not significantly different at any time point. Post therapy injections of IGF-1 on days 4-8 after irradiation lead to a significant increase in amylase-positive acinar cell area which is not significantly different from both unirradiated groups (93-98\% of untreated controls, figure 3). In addition, evaluation of the total amylase protein content in stimulated saliva on day 30 shows that restoration of acinar cell areas in IGF-1 injected mice indeed increase amylase content in stimulated saliva ( $90 \%$ of untreated controls, figure 4 ). In contrast, irradiated mice secrete less amylase (decreased 29\%) compared to unirradiated control saliva samples. The increase in amylase-positive cell area and amount of amylase protein in stimulated saliva on day 30 (figure 4) in irradiated mice receiving IGF-1 correlates with increases in stimulated salivary flow rates on the corresponding time points (figure $2 \mathrm{~A}, \mathrm{~B}$, and $2 \mathrm{C}$ ).

\section{Proliferation indices return to untreated levels in irradiated mice receiving post-therapy IGF-1}

FVB mice exposed to 5 Gy have a 4 -fold increase in PCNA positive salivary acinar cells at days 9 and 30 when compared to untreated controls $(P<0.05$, one-way ANOVA, figure 5A). At days 60 and 90 , irradiated FVB mice continue to show elevated PCNA-positive acinar cell levels that is statistically higher than untreated controls $(P<0.05$, one-way ANOVA). In contrast, the number of PCNA positive acinar cells in irradiated mice injected with IGF-1 gradually decreases from elevated levels (2-3 fold) on days 9 and 30 to levels similar to untreated mice on days 60 and 90 (figure 5A). At later time points (days 60-90), the percentage of PCNA positive acinar cells in mice receiving post-therapy IGF-1 are not statistically different from untreated controls $(P<0.05$, one-way ANOVA). In irradiated mice, the level of PCNA positive ductal cells remains significantly elevated throughout the observation period similar to the profile detected in acinar cells $(P<0.05$, one-way ANOVA), figure 5B). Interestingly, the level of PCNA positive ductal cells in irradiated mice receiving IGF-1 only differs from untreated control mice on day 30. This profile within the ductal cells of irradiated IGF-1 injected mice is strikingly different from the profile of the acinar cells which demonstrate a gradual decrease in PCNA (compare 5A and B).

\section{Discussion}

Radiation treatment of head and neck cancer patients causes significant chronic damage to the salivary glands 

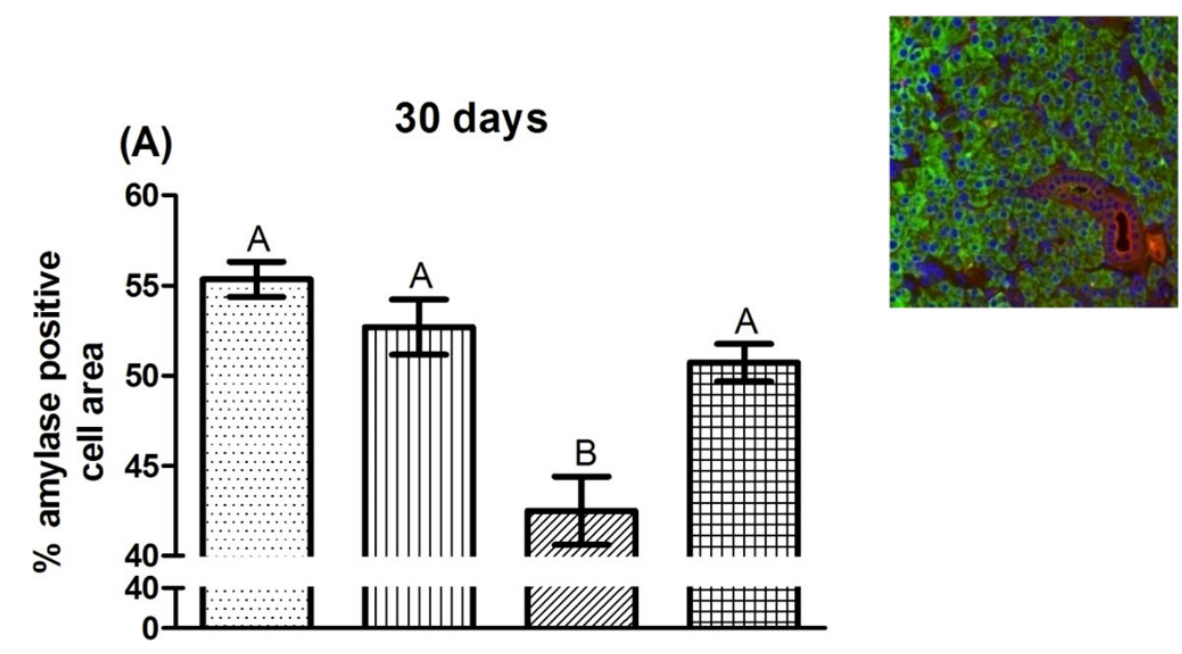

(B) 60 days

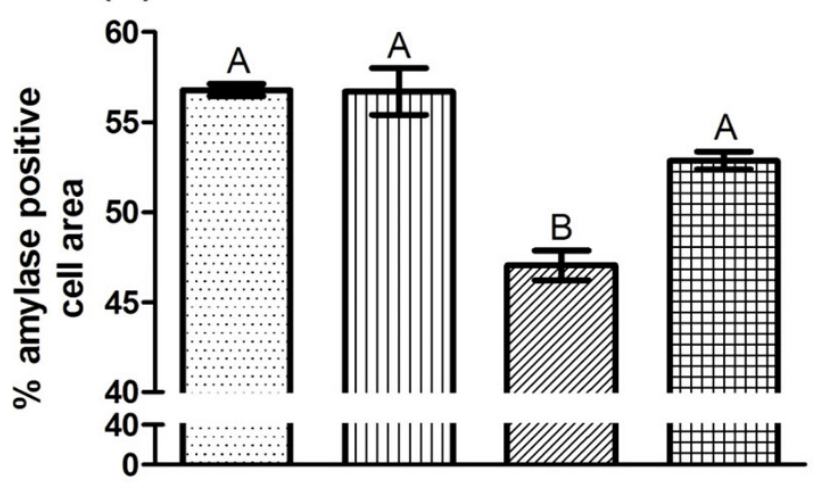

(C) 90 days

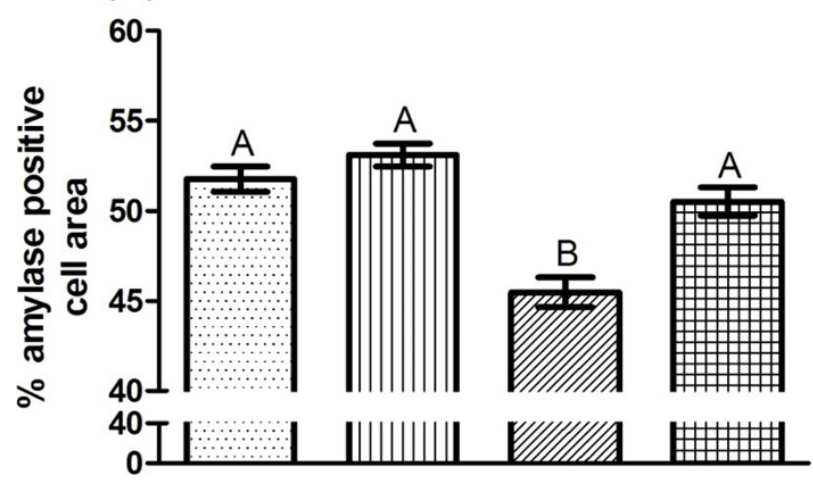

Figure 3 Post therapy IGF-1 restores functional acinar cell area in irradiated mice. The head and neck region of FVB mice was exposed to a single 5 Gy radiation dose and mice received injections of IGF-1 or vehicle on days 4-8 as depicted in figure 1A. Parotid glands were removed on days 30 (A), 60 (B), and 90 (C) after the radiation treatment. Tissues were embedded into paraffin and immunohistochemistry was performed according to the materials and methods section. The insert is a representative image with amylase-positive acinar cells (green), cytokeratinpositive ductal cells (red), and cell nuclei (blue). The graph represents the percentage area of amylase-positive cells in the field of view. All data are composed of the mean and standard error of the mean from 3-8 mice per group. Significant differences $(p<0.05)$ were determined using an ANOVA followed by a post-hoc Bonferroni multiple comparison test. Treatment groups with the same letters are not significantly different from each other within the same day. 


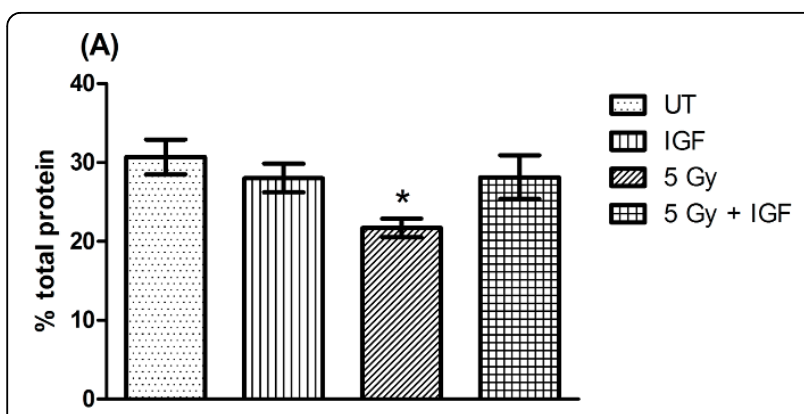

(B)

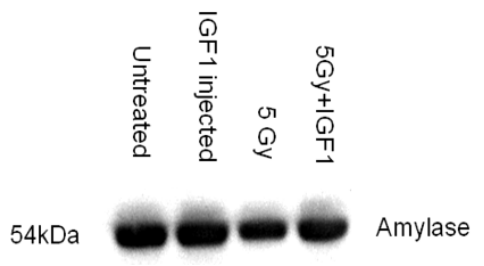

Figure 4 Post therapy IGF-1 restores amylase content in stimulated saliva $\mathbf{3 0}$ days following radiation. The head and neck region of FVB mice was exposed to a single 5 Gy radiation dose and mice received injections of IGF-1 or vehicle on days 4-8 as depicted in figure $1 A$. In (A), stimulated saliva samples from figure 2A were collected and analyzed for total protein content as described in the materials and methods section. The graph represents the percentage of amylase protein (ranging from 50-57 $\mathrm{kD}$ ). All data are composed of the mean and standard error of the mean from 7-8 mice per group. Significant differences $(p<0.05)$ were determined using an ANOVA followed by a Bonferroni test. $\left(^{*}\right)$ in figure $4 \mathrm{~A}$ indicates significant differences between irradiated mice receiving post-therapy IGF-1 or vehicle. A representative Western blot as described in the materials and methods section of day 30 saliva samples is shown in (B).

and there are few therapeutic options available to improve the patients' quality of life. The current findings indicate that post therapy injections of IGF-1 following radiation restore salivary gland function (figure 2), which poses several clinical advantages. Implications include the possibility of restoring salivary gland function in specific patients by delivering an IGF1-type compound to restore deficient salivary gland function, even after radiation therapy.

The reduction in stimulated salivary flow rates for animals receiving a single radiation dose of 5 Gy is significant throughout the 90 day observation period (figure 2). These findings are in agreement with previous studies using single doses of radiation (1-5Gy) [16,19]. Post therapy administration of IGF-1 on days 4-8 after radiation exposure significantly increases salivary flow rates at all time points. On days 60 and 90 flow rates have been restored to untreated levels (figure 2). This further establishes a critical role for IGF-1 in the signaling pathways

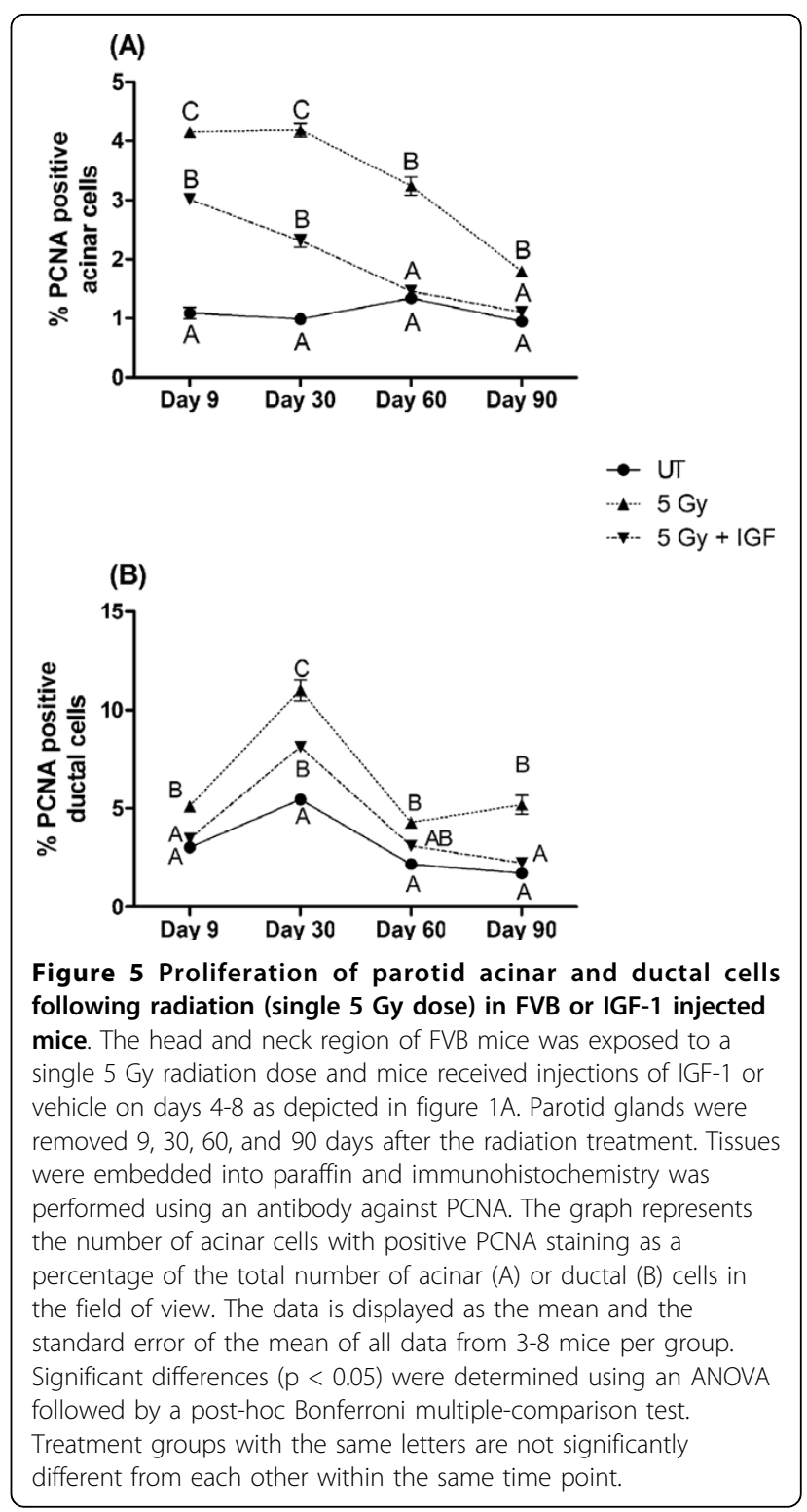

that manage the cellular response to radiation in the salivary glands. It also suggests that post therapy activation of specific downstream molecules may be an effective targeted therapy for the restoration of salivary gland function.

Loss of acinar cells in response to radiation has been widely discussed. While significant levels of apoptosis immediately following radiation have been observed in mice [16,18-20], the presence of apoptosis in irradiated rats has been more variable [28-30]. Because acinar cells contribute a majority of saliva constituents including amylase and water, loss of these cells would impair normal saliva composition and lead to reduction in saliva flow. Our results indicate that reduction of stimulated salivary flow rates as a physiological parameter is closely 
related to reductions in amylase-positive acinar cell area in parotid glands (figure 3). The percentage of amylase positive cells is a useful indicator for the evaluation of actively secreting parotid acinar cells [9]. Our findings further indicate that the loss of amylase positive cells is correlated with a decrease in amylase protein secretion (figure 4). Post therapy administration of IGF-1 to irradiated mice results in significant increases of amylasepositive cells over the course of the observation period compared to irradiated animals that received vehicle alone (figure 3). While irradiated animals have consistently reduced areas of amylase-positive cells on days 30,60 , and 90, administration of IGF-1 after radiation results in increased amylase-positive cells and improved amylase secretion indicative of a restoration of functioning acinar cells (figures 3 and 4). A common observation in salivary glands exposed to radiation is atrophy and loss of glandular weight indicative of cell loss (reviewed in [17]), and our results implicate that functional acinar cell area is restored in animals receiving post therapy IGF-1 injections.

An interesting finding of our study is the influence of post therapy IGF-1 administration on PCNA, a marker of cell proliferation. Tissue homeostasis is maintained by keeping a balance between cell growth and cell death. Salivary glands, unlike other radiosensitive tissues, consist of highly differentiated cells that proliferate slowly (reviewed in (24)). In our study, we observe increased levels of PCNA positive acinar cells in animals receiving a single dose of 5 Gy (figure 5) which could be indicative of compensatory proliferation [31]. In rapidly cycling tissues, compensatory proliferation is a mechanism to replace lost cells; however in slowly cycling tissues, compensatory proliferation correlates with loss of function [31-33]. Our study concurs with these studies in that radiation-induced compensatory proliferation in the salivary gland correlates with reduced physiologic function. It is feasible that the cells actively cycling in the salivary gland have an undifferentiated phenotype that does not produce an appreciable amount of saliva. While the level of PCNA positive acinar cells remains significantly elevated in irradiated animals, mice receiving post therapy IGF-1 decreased the levels of PCNA positive acinar cells to back to control levels on days 60 and 90 (figure 5A). Our study corroborates radiationinduced compensatory proliferation that has been reported in the submandibular salivary glands of mice $[34,35]$ and the parotid salivary glands of rats [36]. Interestingly, administration of pilocarpine prior to radiation treatment of rats resulted in increased compensatory proliferation and preservation of salivary function $[36,37]$. One explanation for the difference between our study and the pilocarpine study is the timing of administration. Our study evaluated alterations in proliferation indices using a post-therapy model (figure 1A) whereas pilocarpine was administered prior to radiation treatment [36].

Currently the salivary gland stem cell is unknown; however, there is growing evidence that progenitor cells are present within the ductal cell network. Immunohistochemical staining of submandibular salivary glands for the stem cell marker c-kit revealed its localization to the excretory duct [25]. In addition, transplantation of salivary cells enriched for c-kit into irradiated mouse salivary glands restores salivary gland function [25]. Our results in irradiated mice demonstrate an increase in PCNA positive ductal cells across the entire time course similar to the acinar cells (figure 5). In contrast, mice receiving post therapy IGF-1 have an increase in PCNA positive ductal cells only at the day 30 time point after radiation treatment. It is possible that IGF-1 may transiently influence the stem/progenitor population within the ductal network in order to restore salivary gland function; however more studies are required to test this hypothesis.

One potential concern for the use of growth factors such as IGF-1 for clinical applications is the effect as a tumor promoter. However, salivary gland tumors were not detected in any of the animals that received five consecutive injections of IGF-1 in our studies. Furthermore, it is more likely that drugable analogs of IGF-1 specific to the tissue or pathways involved would be developed and administered clinically. In addition, the salivary glands could be specifically treated through cannulation of the salivary duct. The long-term translation of this study involves the identification of these pathway specific molecules that could be utilized to specifically target and restore salivary gland function following clinical radiation therapy.

\section{Conclusions}

The results of our study provide further support for the involvement of IGF-1 or analogous compounds in the preservation and restoration of salivary glands following radiation which can lead to diminished side effects and provide head and neck cancer patients with the possibility for improved quality of life.

\section{Abbreviations}

(IGF-1): Insulin-like growth factor-1; (PCNA): proliferating cell nuclear antigen.

\section{Acknowledgements}

The authors wish to thank Dr. Sean Limesand for the use of the Leica DM5500 microscope for imaging. This work was supported in part by $\mathrm{NIH}$ DE18888 and DE16096. The funders had no role in study design, data collection and analysis, decision to publish, or preparation of the manuscript.

\section{Authors' contributions}

OG participated in the study design, carried out physiology experiments, conducted statistical analysis on all data, and drafted the manuscript. JLF 
contributed to the physiology experiments, and completed all amylase analysis experiments. KV completed the proliferation analysis. KHL and RB conceived the studies and participated in design of the experiments. KHL oversaw and coordinated the studies and finalized writing of the manuscript. All authors read and approved the final manuscript.

\section{Competing interests}

$\mathrm{OG}, J L F, K V$, and $\mathrm{RB}$ have nothing to declare. $\mathrm{KHL}$ is listed as an inventor on U.S. Patent Application No. 12/304,359.

Received: 5 February 2010 Accepted: 10 August 2010

Published: 10 August 2010

\section{References}

1. Hancock PJ, Epstein JB, Sadler GR: Oral and dental management related to radiation therapy for head and neck cancer. J Can Dent Assoc 2003, 69:585-590.

2. Nguyen NP, Vos P, Karlsson U, Nguyen P, Dutta S, Lemanski C, Ludin A, Rose S, Nguyen LM, Ward H, Huang S, Sallah S: Quality of life following chemoradiation and postoperative radiation for locally advanced head and neck cancer. ORL J Otorhinolaryngol Relat Spec 2007, 69:271-276.

3. Nguyen NP, Smith HJ, Sallah S: Evaluation and management of swallowing dysfunction following chemoradiation for head and neck cancer. Curr Opin Otolaryngol Head Neck Surg 2007, 15:130-133.

4. Fox PC, van der Ven PF, Sonies BC, Weiffenbach JM, Baum BJ: Xerostomia: evaluation of a symptom with increasing significance. J Am Dent AssoC 1985, 110:519-525

5. Robar JL, Day A, Clancey J, Kelly R, Yewondwossen M, Hollenhorst $H_{\text {, }}$ Rajaraman M, Wilke D: Spatial and dosimetric variability of organs at risk in head-and-neck intensity-modulated radiotherapy. Int J Radiat Oncol Biol Phys 2007, 68:1121-1130.

6. Henson BS, Eisbruch A, D'Hondt E, Ship JA: Two-year longitudinal study of parotid salivary flow rates in head and neck cancer patients receiving unilateral neck parotid-sparing radiotherapy treatment. Oral Oncol 1999 35:234-241.

7. Stephens LC, Ang KK, Schultheiss TE, King GK, Brock WA, Peters LJ: Target cell and mode of radiation injury in rhesus salivary glands. Radiother Oncol 1986, 7:165-174.

8. Turner RJ: Mechanisms of fluid secretion by salivary glands. Ann N Y Acad Sci 1993, 694:24-35.

9. Christensen ME, Hansen HS, Poulsen SS, Bretlau P, Nexo E: Immunohistochemical and quantitative changes in salivary EGF, amylase and haptocorrin following radiotherapy for oral cancer. Acta Otolaryngol 1996, 116:137-143.

10. Stephens LC, King GK, Peters LJ, Ang KK, Schultheiss TE, Jardine JH: Acute and late radiation injury in rhesus monkey parotid glands. Evidence of interphase cell death. Am J Pathol 1986, 124:469-478.

11. Li Y, Taylor JM, Ten Haken RK, Eisbruch A: The impact of dose on parotid salivary recovery in head and neck cancer patients treated with radiation therapy. Int J Radiat Oncol Biol Phys 2007, 67:660-669.

12. Radfar $L$, Sirois DA: Structural and functional injury in minipig salivary glands following fractionated exposure to $70 \mathrm{~Gy}$ of ionizing radiation: an animal model for human radiation-induced salivary gland injury. Oral Surg Oral Med Oral Pathol Oral Radiol Endod 2003, 96:267-274.

13. Rades D, Fehlauer F, Bajrovic A, Mahlmann B, Richter E, Alberti W: Serious adverse effects of amifostine during radiotherapy in head and neck cancer patients. Radiother Oncol 2004, 70:261-264.

14. Cotrim AP, Hyodo F, Matsumoto K, Sowers AL, Cook JA, Baum BJ, Krishna MC, Mitchell JB: Differential radiation protection of salivary glands versus tumor by Tempol with accompanying tissue assessment of Tempol by magnetic resonance imaging. Clin Cancer Res 2007, 13:4928-4933.

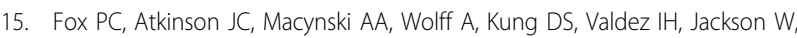
Delapenha RA, Shiroky J, Baum BJ: Pilocarpine treatment of salivary gland hypofunction and dry mouth (xerostomia). Arch Intern Med 1991, 151:1149-1152.

16. Limesand $\mathrm{KH}$, Said S, Anderson SM: Suppression of radiation-induced salivary gland dysfunction by IGF-1. PLOS ONE 2009, 4:e4663.

17. Grundmann O, Mitchell GC, Limesand KH: Sensitivity of salivary glands to radiation: From animal models to therapies. J Dent Res 2009, 88:894-903.
18. Limesand $\mathrm{KH}$, Schwertfeger $\mathrm{KL}$, Anderson SM: MDM2 is required for suppression of apoptosis by activated Akt1 in salivary acinar cells. $\mathrm{Mol}$ Cell Biol 2006, 26:8840-8856.

19. Avila JL, Grundmann O, Burd R, Limesand KH: Radiation-induced salivary gland dysfunction results from p53-dependent apoptosis. Int J Radiat Oncol Biol Phys 2009, 73:523-529.

20. Humphries MJ, Limesand KH, Schneider JC, Nakayama Kl, Anderson SM Reyland ME: Suppression of apoptosis in the PKCdelta null mouse in vivo. J Biol Chem 2006, 281:9728-9737.

21. Limesand $\mathrm{KH}$, Avila JL, Victory $\mathrm{K}$, Chang $\mathrm{H}-\mathrm{H}$, Shin $\mathrm{YJ}$, Grundmann O, Klein R: IGF-1 preserves salivary gland function following fractionated radiation. Int J Radiat. Oncol Biol Phys 2010.

22. Mitchell GC, Fillinger JL, Sittadjody S, Avila JL, Burd R, Limesand KH: IGF1 activates cell cycle arrest following irradiation by reducing binding of $\triangle \mathrm{Np63}$ to the p21 promoter. Cell Death and Disease 2010

23. Lin $A L$, Johnson DA, Wu Y, Wong G, Ebersole JL, Yeh CK: Measuring shortterm gamma-irradiation effects on mouse salivary gland function using a new saliva collection device. Arch Oral Biol 2001, 46:1085-1089.

24. Burgess $\mathrm{KL}$, Dardick I: Cell population changes during atrophy and regeneration of rat parotid gland. Oral Surg Oral Med Oral Pathol Oral Radiol Endod 1998, 85:699-706.

25. Lombaert IM, Brunsting JF, Wierenga PK, Faber H, Stokman MA, Kok T, Visser WH, Kampinga HH, de Haan G, Coppes RP: Rescue of salivary gland function after stem cell transplantation in irradiated glands. PLOS ONE 2008, 3:e2063.

26. Friedrich RE, Bartel-Friedrich $\mathrm{S}$, Holzhausen $\mathrm{HJ}$, Lautenschlager $\mathrm{C}$ : The effect of external fractionated irradiation on the distribution pattern of extracellular matrix proteins in submandibular salivary glands of the rat. J Craniomaxillofac Surg 2002, 30:246-254.

27. Sagowski C, Wenzel S, Tesche S, Jenicke L, Jaehne M: Investigation of radiosialadenitis during fractioned irradiation: sialoscintigraphical and histomorphological findings in rats. Eur Arch Otorhinolaryngol 2003, 260:513-517

28. Boraks G, Tampelini FS, Pereira KF, Chopard RP: Effect of ionizing radiation on rat parotid gland. Braz Dent J 2008, 19:73-76

29. Thula TT, Schultz G, Tran-Son-Tay R, Batich C: Effects of EGF and bFGF on irradiated parotid glands. Ann Biomed Eng 2005, 33:685-695.

30. Paardekooper GM, Cammelli S, Zeilstra LU, Coppes RP, Konings AW: Radiation-induced apoptosis in relation to acute impairment of rat salivary gland function. Int J Radiat Biol 1998, 73:641-648.

31. Denekamp J: Cell kinetics and radiation biology. Int J Radiat Biol Relat Stud Phys Chem Med 1986, 49:357-380

32. Stewart FA, Denekamp J, Hirst DG: Proliferation kinetics of the mouse bladder after irradiation. Cell Tissue Kinet 1980, 13:75-89.

33. Michalowski A: Effects of radiation on normal tissues: hypothetical mechanisms and limitations of in situ assays of clonogenicity. Radiat Environ Biophys 1981, 19:157-172.

34. Bralic M, Muhvic-Urek M, Stemberga V, Golemac M, Jurkovic S, Borcic J, Braut A, Tomac J: Cell death and cell proliferation in mouse submandibular gland during early post-irradiation phase. Acta Med Okayama 2005, 59:153-159.

35. Muhvic-Urek M, Bralic M, Curic S, Pezelj-Ribaric S, Borcic J, Tomac J: Imbalance between apoptosis and proliferation causes late radiation damage of salivary gland in mouse. Physiol Res 2006, 55:89-95.

36. Burlage FR, Faber $H$, Kampinga HH, Langendijk JA, Vissink A, Coppes RP: Enhanced proliferation of acinar and progenitor cells by prophylactic pilocarpine treatment underlies the observed amelioration of radiation injury to parotid glands. Radiother Oncol 2009, 90:253-256.

37. Coppes RP, Vissink A, Zeilstra LJ, Konings AW: Muscarinic receptor stimulation increases tolerance of rat salivary gland function to radiation damage. Int J Radiat Biol 1997, 72:615-625.

Pre-publication history

The pre-publication history for this paper can be accessed here: http://www.biomedcentral.com/1471-2407/10/417/prepub

doi:10.1186/1471-2407-10-417

Cite this article as: Grundmann et al: Restoration of radiation therapyinduced salivary gland dysfunction in mice by post therapy IGF-1 administration. BMC Cancer 2010 10:417. 